\title{
Identification of Orthologous Hemocyanin Subunits in Scorpions: The First Step to Produce a Phylogenetic Tree Using Amino Acid Sequence Data
}

\author{
Hiroaki Sugita', Takao Kuwada', Kensuke Okamura² and Toshiki Makioka' \\ 'Institute of Biological Sciences, University of Tsukuba, Tsukuba, Ibaraki 305-8572, Japan \\ ${ }^{2}$ Master's Program in Biosystem Studies, University of Tsukuba, Tsukuba, Ibaraki 305-8572, Japan
}

(Received 12 March 1998; Accepted 13 July 1998)

\begin{abstract}
Direct protein sequencing of the eight hemocyanin subunits from the scorpion Liocheles australasiae gave the N-terminal sequences for the first 16-41 amino acid residues. From sequence comparisons among $L$. australasiae hemocyanin subunits and between hemocyanin subunits from L. australasiae and Androctonus australis, it is deduced that duplications of two subunits occurred in the lineage of $L$. australasiae. From the $\mathrm{N}$-terminal sequence comparison among hemocyanin subunits from L. australasiae, A. australis, and Buthus sindicus, it is inferred that at least one orthologous subunit derived from a common ancestor is shared among the three scorpions. Thus, $\mathrm{N}$-terminal sequence analysis is regarded as a good method to identify orthologous subunits among many homologues between species.

Key Words: amino acid sequences, evolution, Liocheles australasiae, hemocyanin subunits, orthologous, scorpions.
\end{abstract}

\section{Introduction}

To produce a phylogenetic tree of the evolution of animals using sequence data of protein molecules or genes, we must use orthologous sequences that originated from a sequence in their common ancestor. In the case of chelicerate hemocyanins, which are organized either as hexamers of about $75 \mathrm{kDa}$ subunit chains or as multiples of these hexamers (Van Holde and Miller 1995), the heterogeneity of the monomer subunits has been established by means of polyacrylamide gel electrophoresis (Sugita and Sekiguchi 1975; Markl et al. 1979). The complete amino acid sequences of the subunits have been determined for the hemocyanins from several chelicerates: Japanese horseshoe crab Tachypleus tridentatus (Takagi and Nemoto 1983), American horseshoe crab Limulus polyphemus (Nakashima et al. 1986), a spider, Eurypelma californicum (Schartau et al. 1983; Schneider et al. 1983; Voit and Feldmaier-Fuchs 1990), and a scorpion, Androctonus australis (Buzy et al. 1995). Percentage identities between these sequences range from 53 to 65 (Linzen et al. 1985; Voit and Feldmaier-Fuchs 1990; Beintema et al. 1994; Buzy et al. 1995). All chelicerate hemocyanin subunits completely sequenced to date show a considerable degree of similarity within a species (the spider), between genera (the horseshoe crabs), between orders (the spider and the scorpion), and between classes (the horseshoe crabs and the spider or scorpion). These hemocyanin subunits could not be used for a tree of the animals' evolution, just for evolutionary trees of the hemocyanin molecules (Beintema et al. 1994; Fujimoto et al. 1995; Sugita and 
Shishikura 1995; Burmester and Scheller 1996). To make a phylogenetic tree of chelicerates using hemocyanin molecules, orthologous hemocyanin subunits among species must be identified.

In this paper, the $\mathrm{N}$-terminal amino acid sequences of eight hemocyanin subunits from a scorpion, Liocheles australasiae, are reported and, to identify orthologous subunits on which a phylogenetic tree can be based, they are compared with the $\mathrm{N}$-terminal sequences of seven hemocyanin subunits from the scorpion Androctonus australis (Jollès et al. 1979). The evolutionary implication of the existence of many homologous sequences in species is discussed.

\section{Materials and Methods}

Liocheles australasiae was obtained from Iriomote Island, the Ryukyu Islands, Japan. Hemolymph was collected by puncturing the heart with a micro-syringe and kept with a roughly equal volume of glycerin at $-20^{\circ} \mathrm{C}$.

Polyacrylamide disc and slab gels at $\mathrm{pH} 8.9$ were prepared according to the method of Davis (1964), and to distinguish a dimer subunit from monomer subunits of hemocyanin, the acrylamide concentration was varied from 5 to $10 \%$ by changing the volume ratio of acrylamide solution to water (Sugita and Sekiguchi 1975). The hemocyanin subunit bands in a 7.5\% disc gel were cut out and the subunits in the bands were re-electrophoresed in to a $7.5 \%$ slab gel.

The subunits in the slab gel were transferred to a polyvinylidene difluoride (PVDF) sheet which had been activated for $1 \mathrm{~min}$ in $100 \%$ methanol and soaked in transfer buffer. The transfer buffer contained $25 \mathrm{mM}$ Tris, $192 \mathrm{mM}$ glycine, $\mathrm{pH} 8.3,4 \%$ methanol, and $0.02 \%$ sodium dodecyl sulfate. Electrophoretic transfer was carried out for $6 \mathrm{hr}$ at $2.4 \mathrm{~mA} / \mathrm{cm}^{2}$ in a blotting apparatus (Towbin et al. 1979). The PVDF sheet containing the hemocyanin subunits was stained for 5 minutes with a Coomassie blue solution ( $0.1 \%$ in $42 \%$ methanol $/ 17 \%$ acetic acid) and destained for a few minutes with $90 \%$ methanol, washed with distilled water, and then dried overnight.

The protein spots stained were cut out from the PVDF sheet and mounted in the reaction chamber of a protein sequencer. $\mathrm{N}$-terminal sequence analysis was performed with a Procise 492 gas phase sequencer (Applied Biosystems).

\section{Results and Discussion}

Electrophoretic patterns of $L$. australasiae hemocyanin in various acrylamide concentrations from 5 to $10 \%$ are shown in Fig. 1, and the relation between the relative mobility of the protein bands and the acrylamide concentration is shown in Fig. 2. If two molecules have different net charges but a similar molecular size, such plots as in Fig. 2 yield parallel lines (Sugita and Sekiguchi 1975). From the results in Fig. 2, it is apparent that hemocyanin subunits LA1-7 have a molecular size similar to that of bovine serum albumin $(67 \mathrm{kDa})$ and that LA8 has a molecular size definitely different from these subunits and nearly equal to the dimer size of bovine serum albumin.

Direct protein sequencing of the seven hemocyanin monomer and one dimer 


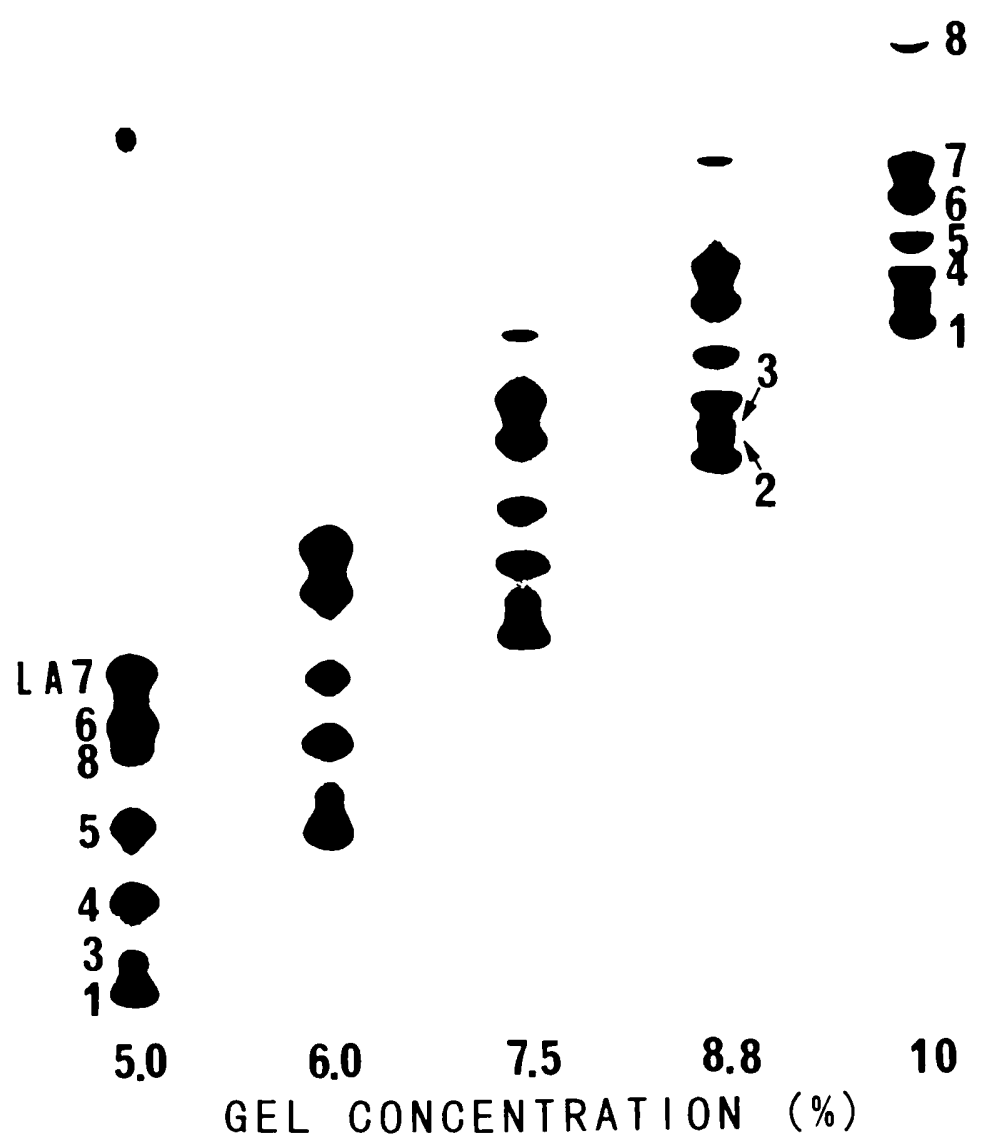

Fig. 1. Electrophoretic patterns of $L$. australasiae hemocyanin subunits in various acrylamide gel concentrations. Hemocyanin subunit bands in 7.5\% acrylamide gel were named LA1-8 in order of mobility.

subunits transferred to the PVDF sheet gave the sequences for the first $16-41$ amino acid residues as shown in Fig. 3. Like most chelicerate hemocyanin subunits, the subunits of $L$. australasiae hemocyanin possessed lysine-glutamine and leucinephenylalanine residues in positions 5-6 and 12-13, respectively. When the N-terminal sequences of $L$. australasiae hemocyanin subunits are compared within the species, it is evident that there are two pairs of similar subunits showing sequence differences of $0.13 \%$ (Table 1). Pairwise distances between the subunits exclusive of these two pairs range from $41 \%$ to $74 \%$. This range is wider than that of pairwise distances of 35-47\% (Beintema et al. 1994; Buzy et al. 1995; Linzen $e$ t al. 1985; Voit and FeldmaierFuchs 1990) between complete amino acid sequences of chelicerate hemocyanins. It seems that the big pairwise distance scores between $L$. australasiae hemocyanin subunits in Table 1 are related to the strong conservation of functional amino acids in the second domain, which contains the binuclear copper oxygen-binding site. Each of the two pairs of similar sequences, LA1-LA2 and LA3-LA4, shows similar difference scores for each of the seven subunits from A. australis (Table 2). For example, 


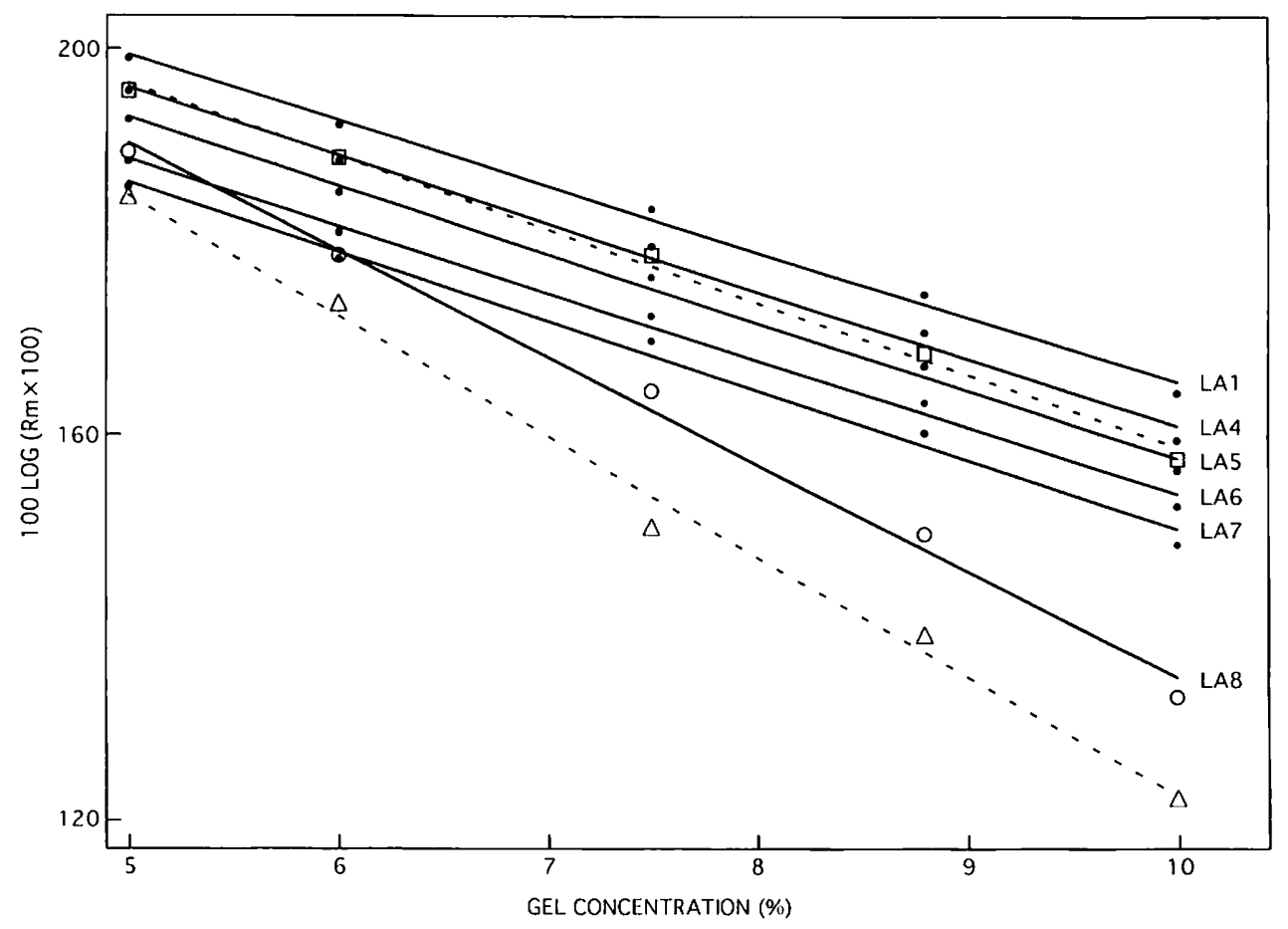

Fig. 2. The effect of various gel concentrations on the mobility of hemocyanin monomer and dimer subunits. The subunits LA2 and LA3 were separated between LA1 and LA4 and their slopes were almost equal to those of LA1 and LA4. $\bullet$, hemocyanin monomer; O,hemocyanin dimer; $\square$, bovine serum albumin monomer; $\triangle$, bovine serum albumin dimer.
L A 1
1 'TVEKQAKL'LPI
10
30
40
LA 2 TVQEKQAKLLPLFQPK
L A 3
TVKEKQQRVVELFQHLTALTEEHIKP
L A 4
TVKEKQQRVVELFQHLTALTEEHIKPED-RDPRLAGVGILR
L A 5
$L \wedge 6$
L A 7
VLHEKQIR I LNLFKHLSVATVGGGVSKEQRDPRLXAHVVGV
L A 8
TLEEKQNKVLPLFRHLTSLTRTQL-PGELRDPRLKXVY
GVQDKQKRLLPLFKHLTSLTTEQL-PVDERDSRLKKVGVL
TADVAEKQKRVIPLFQFVVLTTRQKF-SRAEKDAFRDL

Fig. 3. N-terminal amino acid sequences of hemocyanin subunits (LA1-8) from L. australasiae. Undetermined residues are indicated by $\mathrm{X}$. Dashes represent gaps introduced under the necessity of comparing all $\mathrm{N}$-terminal sequences.

the distance between LA1 and AA2 is equal to that between LA2 and AA2. The distance score of $25 \%$ between LA1 and AA6 is smaller than that of $44 \%$ between LA2 and AA6. However, the different residues in the first 16 sites between LA1 and AA 6 come to 6 , that is, $38 \%$. Therefore, this large difference between $25 \%$ and $44 \%$ is caused by the large difference in sequence lengths of LA 1 and LA2. In acrylamide gel electrophoresis, moreover, L. australasiae hemocyanin subunits did not show any 
Table 1. N-terminal \% difference scores among $L$. australasiae hemocyanin subunits (LA1-8).

\begin{tabular}{ccccccccc}
\hline & LA 1 & LA2 & LA3 & LA4 & LA5 & LA6 & LA7 & LA8 \\
\hline LA1 & & 13 & 50 & 43 & 68 & 42 & 41 & 66 \\
LA2 & $2 / 16$ & & 50 & 50 & 63 & 44 & 44 & 50 \\
LA3 & $14 / 26$ & $8 / 16$ & & 0 & 65 & 50 & 50 & 62 \\
LA4 & $18 / 42$ & $8 / 16$ & $0 / 26$ & & 65 & 47 & 48 & 69 \\
LA5 & $27 / 40$ & $10 / 16$ & $17 / 26$ & $26 / 40$ & & 56 & 59 & 74 \\
LA6 & $15 / 36$ & $7 / 16$ & $13 / 26$ & $17 / 36$ & $20 / 36$ & & 42 & 71 \\
LA7 & $16 / 39$ & $7 / 16$ & $13 / 26$ & $19 / 40$ & $23 / 39$ & $15 / 36$ & & 69 \\
LA8 & $23 / 35$ & $8 / 16$ & $16 / 26$ & $24 / 35$ & $25 / 34$ & $24 / 34$ & $24 / 35$ & \\
\hline
\end{tabular}

Values in the upper right half of the table are the percent differences between $\mathrm{N}$-terminal amino acid sequences. Values in the lower left half of the table are different residues/compared residues in pairwise sequence comparisons. In pairwise comparisons, gaps were inserted to minimize the difference scores.

polymorphism. Thus, it is thought that duplications of two subunits probably gave rise to the two pairs in the lineage of L. australasiae.

In arthropod hemocyanins, the subunits associate noncovalently in most cases, although a few hemocyanins contain one or more disulfide-linked dimers. The disulfide-bridged unit bonds hexamers together, and in some cases unusually strong noncovalent interactions suffice to cement hexamers together (Van Holde and Miller 1995). Direct protein sequencing of the subunit LA8 gave a sequence, but no signal for another sequence. Therefore, LA8 is either a noncovalently cemented homodimer or LA8 is a disulfide-linked homo- or heterodimer. This matter will be resolved by a disulfide bond reduction experiment.

When the $\mathrm{N}$-terminal sequences of hemocyanin subunits are compared between L. australasiae and $A$. australis, the sequence difference between LA5 and AA3B is $8 \%$ (Table 2). In comparison with the two pairs of similar subunits within $L$. australasiae, this difference score is small enough to use for evolutionary studies of the divergence of these two scorpion species. It seems that LA5 and AA3B are orthologous subunits that originated from a subunit found in their common ancestor. Furthermore, there is a considerable likelihood from the sequence difference scores and similar traits in the N-terminals that LA7 and AA5A as well as LA3-LA4 and AA 4 are orthologous. In a strict sense of the word "orthologous", one of LA3 and LA4 is orthologous to AA4, but we can use either of them to study the divergence pattern and time of splitting of $L$. australasiae and $A$. australis, if a duplication introduced LA 3 and LA4 into the $L$. australasiae lineage after the divergence of these two scorpion lineages. Thus, $\mathrm{N}$-terminal sequence analysis is regarded as a good method to identify orthologous subunits among many homologues between species.

Recently Ali et al. (1995) analyzed two N-terminal sequences out of seven hemocyanin subunits from the scorpion Buthus sindicus. A subunit named Bsin 3 has the N-terminal sequence "VLHEKQIRXXQLFD" for the first 14 positions. The sequence difference between Bsin 3 and LA5 is about $17 \%$ and the hemocyanin subunits of Bsin3, LA5, and AA3B show similar traits in the $\mathrm{N}$-terminal sequences. From the relation between $\mathrm{N}$-terminal sequences, we infer that the three subunits are orthologous and thus available for making a phylogenetic tree of these scorpions. Therefore, we may produce a phylogenetic tree using the complete amino acid 


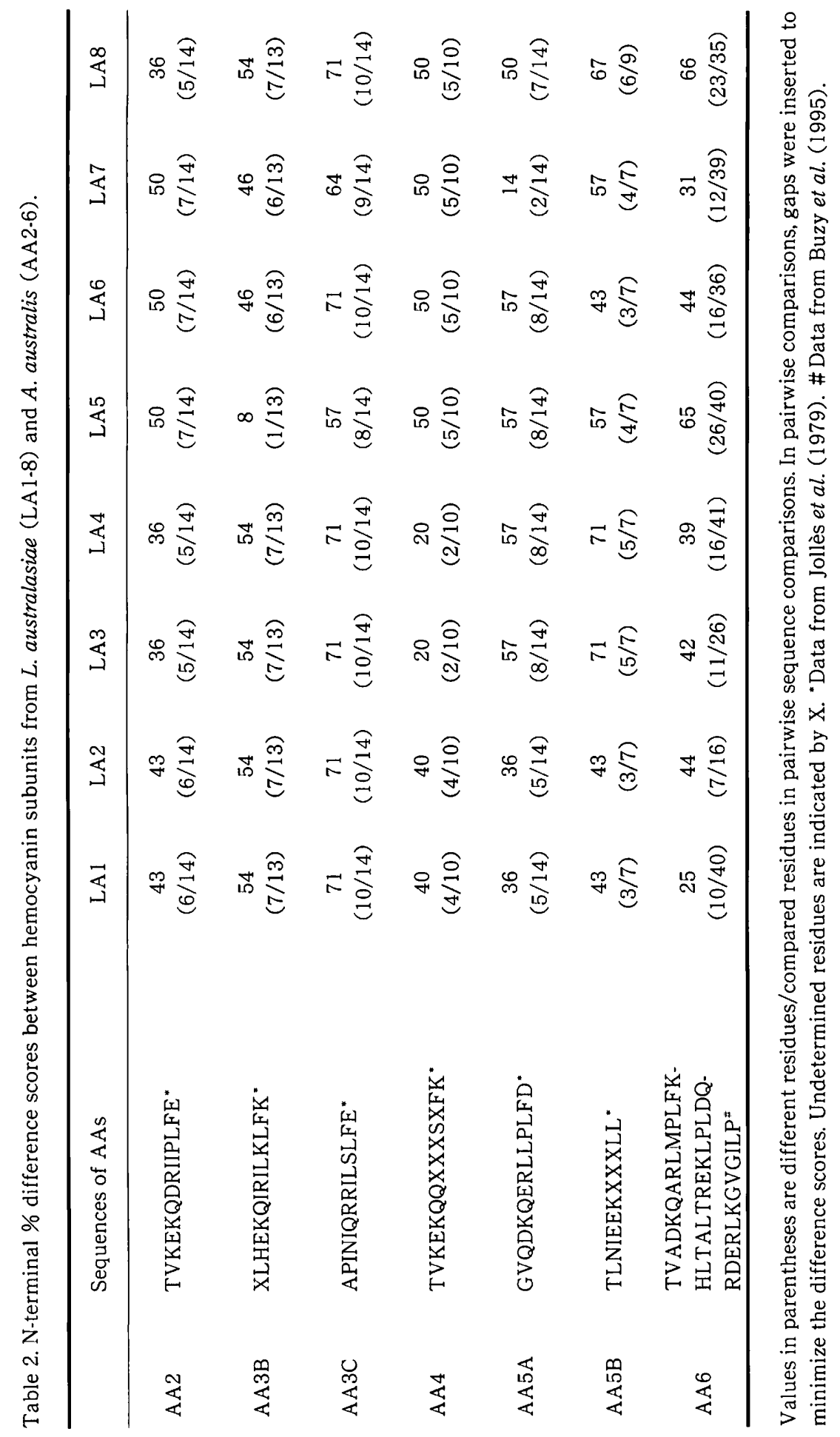


sequences of the orthologous hemocyanin subunits identified by $\mathrm{N}$-terminal sequence comparison between animal species.

\section{Acknowledgments}

We thank Mrs. H. Kondo for amino acid sequence analysis and Dr. K. Yahata and Mr. K. Yamazaki and Mr. T. Takayama for collecting L. australasiae.

\section{References}

Ali, S. A., Zaidi, Z. H. and Abbasi, A. 1995. Oxygen transport proteins: I. Structure and organization of hemocyanin from scorpion (Buthus sindicus). Comparative Biochemistry and Physiology 112A: 225-232.

Beintema, J. J., Stam, W. T., Hazes, B. and Smidt, M. P. 1994. Evolution of arthropod hemocyanins and insect storage proteins (hexamerins). Molecular Biology and Evolution 11: 493-503.

Burmester, T. and Scheller, K. 1996. Common origin of arthropod tyrosinase, arthropod hemocyanin, insect hexamerin, and dipteran arylphorin receptor. Journal of Molecular Evolution 42: 713-728.

Buzy, A., Gagnon, J., Lamy, J., Thibault, P., Forest, E. and Hudry-Clergeon, G. 1995. Complete amino acid sequence of the Aa6 subunit of the scorpion Androctonus australis hemocyanin determined by Edman degradation and mass spectrometry. European Journal of Biochemistry 233: 93-101.

Davis, B. J. 1964. Disc electrophoresis-II. Method and application to human serum proteins. Annals of the New York Academy of Sciences 121: 404-427.

Fujimoto, K., Okino, N., Kawabata, S., Iwanaga, S. and Ohnishi, E. 1995. Nucleotide sequence of the cDNA encoding the proenzyme of phenol oxidase $\mathrm{A}_{1}$ of Drosophila melanogaster. Proceedings of the National Academy of Sciences of the United States of America 92: 7769-7773.

Jollès, J., Jollès, P., Lamy, J. and Lamy, J. 1979. Structural characterization of seven different subunits in Androctonus australis haemocyanin. FEBS Letters 106: 289-291.

Linzen, B., Soeter, N. M., Riggs, A. F., Schneider, H.J., Schartau, W., Moore, M. D., Yokota, E., Behrens, P. Q., Nakashima, H., Takagi, T., Nemoto, T., Vereijken, J. M., Bak, H. J., Beintema, J. J., Volbeda, A., Gaykema, W. P. J. and Hol, W. G. J. 1985. The structure of arthropod hemocyanins. Science 229: 519-524.

Markl, J., Markl, A., Schartau, W. and Linzen, B. 1979. Subunit heterogeneity in arthropod hemocyanins: I. Chelicerata. Journal of Comparative Physiology 130: 283-292.

Nakashima, H., Behrens, P. Q., Moore, M. D., Yokota, E. and Riggs, A. F. 1986. Structure of hemocyanin II from the horseshoe crab, Limulus polyphemus. Sequences of the overlapping peptides, ordering the $\mathrm{CNBr}$ fragments, and the complete amino acid sequence. Journal of Biological Chemistry 261: 10526-10533.

Schartau, W., Eyerle, F., Reisinger, P., Geisert, H., Storz, H. and Linzen, B. 1983. Hemocyanins in spiders, XIX. Complete amino-acid sequence of subunit $d$ from Eurypelma californicum hemocyanin, and comparison to chain $e$. Hoppe-Seyler's Zeitschrift für Physiologische Chemie 364: 1383-1409.

Schneider, H.-J., Drexel, R., Feldmaier, G., Linzen, B. Lottspeich, F. and Henschen, A. 1983. Hemocyanins in spiders, XVIII. Complete amino-acid sequence of subunit $e$ from Eurypelma 
califormicum hemocyanin. Hoppe-Seyler's Zeitschrift für Physiologische Chemie 364: 1357-1381.

Sugita, H. and Sekiguchi, K. 1975. Heterogeneity of the minimum functional unit of hemocyanins from the spider (Argiope bruennichii), the scorpion (Heterometrus sp.), and the horseshoe crab (Tachypleus tridentatus). Journal of Biochemistry 78: 713-718.

Sugita, H. and Shishikura, F. 1995. A case of orthologous sequences of hemocyanin subunits for an evolutionary study of horseshoe crabs: Amino acid sequence comparison of immunologically identical subunits of Carcinoscorpius rotundicauda and Tachypleus tridentatus. Zoological Science 12: 661-667.

Takagi, T. and Nemoto, T. 1983. Primary structure and copper binding sites of hemocyanin. Zoological Magazine 92: 540.

Towbin, H., Staehelin, T. and Gordon, J. 1979. Electrophoretic transfer of proteins from polyacrylamide gels to nitrocellulose sheets: Procedure and some applications. Proceedings of the National Academy of Sciences of the United States of America 76: 4350-4354.

Van Holde, K. E. and Miller, K. I. 1995. Hemocyanins. Advances in Protein Chemistry 47: 1-81.

Voit, R. and Feldmaier-Fuchs, G. 1990. Arthropod hemocyanins. Molecular cloning and sequencing of cDNAs encoding the tarantula hemocyanin subunits a and e. Journal of Biological Chemistry 265: 19447-19452. 